\title{
THE CROSSING NUMBERS OF JOIN OF SOME GRAPHS WITH $n$ ISOLATED VERTICES
}

\author{
ZongPeng Ding And YuAnQIu HuAng ${ }^{1}$ \\ College of Mathematics and Computer Science \\ Hunan Normal University \\ Changsha, Hunan 410081, P.R. China \\ e-mail: dzppxl@163.com \\ hyqq@hunnu.edu.cn
}

\begin{abstract}
There are only few results concerning crossing numbers of join of some graphs. In this paper, for some graphs on five vertices, we give the crossing numbers of its join with $n$ isolated vertices.
\end{abstract}

Keywords: disconnected graph, crossing number, join product.

2010 Mathematics Subject Classification: 05C10, 05C38.

\section{REFERENCES}

[1] K. Asano, The crossing number of $K_{1,3, n}$ and $K_{2,3, n}$, J. Graph Theory 10 (1986) $1-8$. doi:10.1002/jgt.3190100102

[2] J.A. Bondy, U.S.R. Murty, Graph Theory with Applications (North-Holland, New York-Amsterdam-Oxford, 1982).

[3] P. Erdős and R.K. Guy, Crossing number problems, Amer. Math. Monthly 80 (1973) $52-58$. doi: $10.2307 / 2319261$

[4] P.T. Ho, On the crossing number of $K_{1, m, n}$, Discrete Math. 308 (2008) 5996-6002. doi:10.1016/j.disc.2007.11.023

[5] Y. Huang and T. Zhao, The crossing number of $K_{1,4, n}$, Discrete Math. 308 (2008) 1634-1638. doi:10.1016/j.disc.2006.12.002

\footnotetext{
${ }^{1}$ This work is supported by the National Natural Science Foundation of China (Grant Nos. $11301169 \& 11371133)$ and Y. Huang is the corresponding author.
} 
[6] D.J. Kleitman, The crossing number of $K_{5, n}$, J. Combin. Theory 9 (1970) 315-323. doi:10.1016/S0021-9800(70)80087-4

[7] M. Klešč, The join of graphs and crossing numbers, Electron. Notes Discrete Math. 28 (2007) 349-355.

doi:10.1016/j.endm.2007.01.049

[8] K. Zarankiewicz, On a problem of P. Turán concerning graphs, Fund. Math. 41 (1955) 137-145.

doi:10.4064/fm-41-1-137-145

Received 31 October 2016

Revised 2 March 2017

Accepted 2 March 2017 